\title{
Stage of Breast Cancer Screening Adoption; The Role of Cognitive Predictors
}

\author{
Ebrahim Naimi ${ }^{1}$, Mehdi Mirzaei-Alavijeh ${ }^{2}$, Touraj Ahmadi Jouybari ${ }^{3}$, Mari \\ Ataee $^{3}$, Abbas Aghaei ${ }^{4}$, Hadi Ahmadi Jouybari5*
}

\begin{abstract}
Breast cancer is the most common cancer among women. The present study investigated the stage of breast cancer screening adoption and the role of some of the cognitive predictors in a sample of Iranian married women. There were a total of 334 married women clients of eight health centers in Kermanshah city, the west of Iran, were randomly selected to participate voluntarily. Women filled out a self-report questionnaire. Data were analyzed by SPSS version 20 using One-way ANOVA, $\chi 2$-test, Fisher's exact test, and bivariate correlations statistical tests at 95\% significant level. The mean age was 39.75 years [SD: 7.73 ]. Nearly $58.4 \%, 26.9 \%, 3 \%, 9.6 \%$, and $2.1 \%$ of the respondents were reported pre-contemplation, contemplation, preparation, action, and maintenance of breast cancer screening adoption, respectively. There was a significant association between age $(\mathrm{P}=0.005)$, and positive family history of breast cancer $(\mathrm{P}=0.037)$, perceived susceptibility $(\mathrm{P}=0.005)$, perceived severity $(\mathrm{P}=0.001)$ and knowledge of symptoms $(\mathrm{P}=0.018)$ with breast cancer stage of screening adoption. Our findings can provide better knowledge for the development and implementing of stage-targeted breast cancer screening adoption promotion programs. We conclude that it seems that the focus of health planner should be to rise to perceived severity; perceived susceptibility and knowledge of symptoms of breast cancer about the promotion of breast cancer screening adoption an Iranian woman.
\end{abstract}

Keywords: Cancer- health programs planning woman

Asian Pac J Cancer Prev, 20 (8), 2373-2378

\section{Introduction}

Cancer has been known as the third cause of death after cardiovascular diseases and accidents in Iran (Saadat, et al., 2015). Breast cancer is the most common cancer among women worldwide (Ibrahim et al., 2017). Breast cancer in Iran has been reported moderately so that the spread of that is 25 to 30 per 100,000 population, and 7,000 to 9,000 new cases are detected every year in Iran (Dafei et al., 2017). Diagnosis and treatment of breast cancer have significant effects on the physical, psychological, and social health of patients (Albert et al., 2004). Aggressive and long-term treatments due to late detection of the breast cancer cause psychosocial distress and reduce the life quality of patients (Parkin et al., 2001). Breast cancer is one of the main challenges in health policies in developing countries such as Iran, and despite the technological advances in diagnostic and therapeutic methods, there has been an increase in the incidence and mortality due to breast cancer in Iran, which results from late diagnosis especially at advanced stages of the disease (Dafei et al., 2017). Early diagnosis of the disease is important in improving the outcomes and survival rate and survival of the patient (Giordano et al., 2004). In the case of early detection, timely treatment, and appropriate treatment, the chance of recovery will be increased and life expectancy will increase in more than $90 \%$ of patients (Parkin et al., 2001). However, a significant proportion of women are not diagnosed at an early stage (Harding et al., 2015). Knowledge increasing is one of the best ways to reduce the risk of cancer mortality (Pennypacker et al., 1999). Several studies on health behaviors emphasize that comprehensive prevention programs need to identify the determinants of individual and environmental behaviors influencing health behaviors; meanwhile, it is more important to identify influential and changeable cognitive predictors (Kok et al., 2016). Personality traits and cognitive determinants can make people susceptible to disease or uptake and maintenance of healthy behaviors (Hosseini et al., 2016; Mirzaei-Alavijeh et al., 2014; Jalilian et al, 2014). Although the factors associated with multi-faceted breast cancer screening behaviors are known

${ }^{1}$ Department of Public Health, Faculty of Health, Yasuj University of Medical Sciences, Yasuj, ${ }^{2}$ Social Development and Health Promotion Research Center, Health Institute, ${ }^{3}$ Clinical Research Development Center, Imam Khomeini Hospital, Kermanshah University of Medical Sciences, Kermanshah, ${ }^{4}$ Social Determinants of Health Research Center, Research Institute for Health Development, Kurdistan University of Medical Sciences, Kurdistan, ${ }^{5}$ Infectious Disease and Tropical Medicine Center, Health Research Institute, Babol University of Medical Science, Babol, Iran.*For Correspondence: hadiaj1397@gmail.com 
to be multidimensional, people's awareness is an essential component in coping and preventing breast cancer, and one of the strategies of the world health organization in the cancer control program has been to raise awareness (Royseand Dignan, 2009). Moreover, Projaska, and Diclemente in their Transtheoretical Model present a concept called stage of change and mention that people at different levels are motivated and ready for performing the behavior. These behavioral stages and levels include five distinguished stages: (a) Precontemplation (people do not intend to take action in the foreseeable future, for example in the next 6 months); (b) Contemplation (people are intending to start healthy behavior in the foreseeable future); (d) Preparation (people are ready to take action within the next 30 days); (c) Action (people have recently changed their behavior); (e) Maintenance (people have sustained their behavior change for a while); therefore, different educational interventions can be utilized considering each level (Prochaska and Velicer, 1997). The present study investigated the stage of breast cancer screening adoption and the role of some of cognitive predictors in a sample of Iranian married women.

\section{Materials and Methods}

\section{Procedures and participant}

There were a total of 334 married women clients of eight health centers, in Kermanshah city, the west of Iran, were randomly selected to participate voluntarily, during 2016. To participation and data gathering; First, different areas of the study based on the division of the geographical region were classified, a total of eight health centers were randomly selected from social class. Then, women referred to the health centers, voluntarily selected and informed about study purpose to participate in this study. Finally, the volunteers filled out the questionnaire. The stage of breast cancer screening adoption of participants was classified using the 5 stages of change items and the stages compared with cognitive predictors. Only the subjects aged 30 to 70 years old who were married were eligible to participate in this study. This research has been approved by the Research Ethics Committees (REC) at the Kermanshah University of medical sciences (KUMS. REC.1395.277).

\section{Tools}

A pilot study was conducted prior to conducting the main project among 30 women, similar to those who participated in the main study, to assess the utility of the instrumentation. Questionnaire included three sections, a) demographic determinants, b) cognitive predictors of breast cancer (knowledge about symptoms of breast cancer, perceived susceptibility and perceived severity), and c) stages of change items.

\section{Demographic Determinants}

Age (year), educational level (primary school, secondary school, high school, academic), job (housewife, employed), family history of breast cancer (yes, no), and economic status (poor, average, rich).

\section{Cognitive Predictors}

Cognitive Predictors questionnaire was designed based on a standard questionnaire (Griva et al., 2013; Lopez-McKee, 2010; Wang et al., 2014) and included 17 items under three constructs including (a) perceived susceptibility; (b) perceived severity; and (c) knowledge of breast cancer symptoms. Four items evaluated perceived susceptibility about breast cancer (e.g., "I will not get complications of breast cancer"). Six items were designed to evaluate perceived severity about breast cancer (e.g., "I think that breast cancer is a dangerous disease"). Knowledge of breast cancer symptoms were measured by a 7-item. Examples: painless tumors, mastitis and skin thickening breast. Responses to items were either yes, or no or. Moreover, in order to facilitate participants' responses to the items, perceived susceptibility and perceived severity items were standardized to a 5-point Likert scale, ranging from 1 (strongly disagree) to 5 (strongly agree). Reliability coefficients for each section of questionnaire were as follows: perceived severity $(\alpha=0.86)$; perceived susceptibility ( $\alpha=0.63$ ); and knowledge of breast cancer symptoms $(\alpha=0.75)$.

\section{Stage of Change}

Stage of breast cancer screening behaviors was designed based on a standard scale (Spencer et al., 2005). Participates were classified into five stages: pre-contemplation (not thinking about screening adoption in next year), contemplation thinking about screening adoption in next year), preparation (had plan to screening adoption in next month), action (perform screening in past 14 months and are planning to perform another one in next year), and maintenance (perform 2 or more screening on schedule and planning to perform another one within the next year).

\section{Statistical Analysis}

Quantitative variables were expressed as means with $\mathrm{SD}$, and qualitative/categorical ones as frequencies and percentages. One-way ANOVA and Fisher's exact test were performed to evaluate the association between stage of change with demographic determinants, perceived susceptibility, perceived severity, and knowledge of symptoms. In addition, bivariate correlations were computed to ascertain the magnitude and direction of the associations between the perceived susceptibility, perceived severity and knowledge of symptoms scores. Cronbach's Coefficient Alpha was used to estimate the internal consistency of the perceived susceptibility and perceived severity measures. Split half was used to estimate the internal consistency of the knowledge of breast cancer symptoms measures. Furthermore, at least 10 faculty members and qualified individuals were consulted to determine the validity of the questionnaire and face and content validity were determined. The level of significance was $<0.05$. Data were analyzed by the SPSS software for Windows, ver. 20.0.

\section{Results}

The mean age of women was 39.75 years [SD: 
Table 1. Distribution of the Socio- Demographic Determinants among Women

\begin{tabular}{lcc}
\hline Variables & Number & Percent \\
\hline Age groups & & \\
$30-40$ & 203 & 60.8 \\
$41-50$ & 94 & 28.1 \\
$51-60$ & 37 & 11.1 \\
Educational level & & \\
Under Diploma & 197 & 59.0 \\
Diploma & 94 & 28.1 \\
Academic & 43 & 12.9 \\
Job & & \\
Employed & 21 & 6.3 \\
Housewife & 313 & 93.7 \\
Positive history of breast cancer in family & \\
Yes & 10 & 3.0 \\
No & 324 & 97.0 \\
Economic Status & & \\
Poor & 83 & 24.9 \\
Average & 188 & 56.3 \\
Good & 63 & 18.9 \\
\hline
\end{tabular}

7.73]. Nearly $58.4 \%, 26.9 \%, 3 \%, 9.6 \%$, and $2.1 \%$ of the respondents were in pre-contemplation, contemplation, preparation, action, and maintenance about breast cancer screening adoption, respectively. More details of the demographic determinants of the participants are shown in Table 1.

Table 2 shows Mean, standard deviation (SD) and bivariate correlations between the cognitive predictors. Perceived susceptibility about breast cancer was
Table 2. Correlations between the Cognitive Predictors

\begin{tabular}{|c|c|c|c|c|}
\hline & Mean (SD) & $\begin{array}{l}\text { Score } \\
\text { Range }\end{array}$ & $\begin{array}{c}\text { Perceived } \\
\text { Susceptibility }\end{array}$ & $\begin{array}{c}\text { Perceived } \\
\text { Severity }\end{array}$ \\
\hline $\begin{array}{l}\text { Perceived } \\
\text { Susceptibility }\end{array}$ & $12.87(3.1)$ & $4-20$ & & \\
\hline $\begin{array}{l}\text { Perceived } \\
\text { Severity }\end{array}$ & $22.61(4.84)$ & $6-30$ & $0.150 * *$ & 1 \\
\hline $\begin{array}{l}\text { Knowledge } \\
\text { of symptoms }\end{array}$ & $2.11(2.01)$ & $0-7$ & $0.201 * *$ & 0.065 \\
\hline
\end{tabular}

** Correlation is significant at the 0.01 level (2-Tailed).

associated with the perceived severity of breast cancer $(\mathrm{r}=0.150)$. In addition, knowledge of symptoms of breast cancer was associated with the perceived susceptibility about breast cancer $(r=0.201)$. Moreover, no significant association between knowledge of symptoms and perceived severity of breast cancer $(\mathrm{r}=0.065)$.

Classification of demographic and cognitive predictors by breast cancer stage of screening adoption was shown in Table 3. We found that women in stages of action or maintenance of breast cancer screening adoption have older age $(\mathrm{P}=0.005)$. In addition, there was a significant association between having a family history of breast cancer with breast cancer stage of screening adoption $(\mathrm{P}=0.037)$. However, there was no significant difference between educational level and job, economic status, and menopause with breast cancer stage of screening adoption. Moreover, there was a significant association between susceptibility $(\mathrm{P}=0.005)$, severity $(\mathrm{P}=0.001)$ and knowledge of symptoms $(\mathrm{P}=0.018)$ with the stage of screening adoption.

The descriptive explanations of knowledge of symptoms items are shown in Table 4. The mean score of knowledge about breast cancer symptoms was $2.11[95 \%$ CI: $1.89,2.32]$, ranged from 0 to 7 . Our results showed,

Table 3. Classification of Demographic and Cognitive Predictors by Stage of Screening Adoption

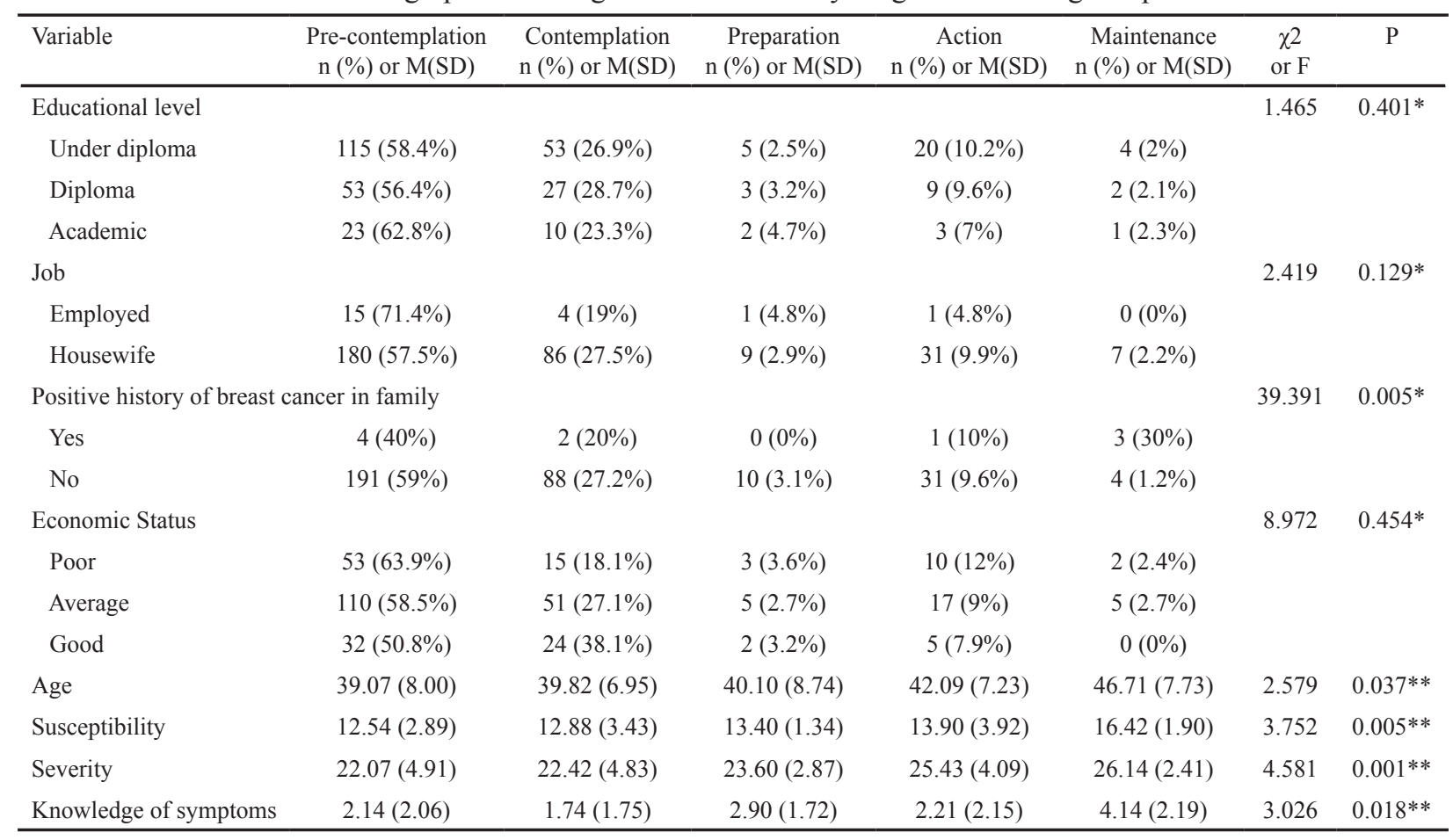

*, Fisher's exact test; **, One-way ANOVA 
Table 4. Women Response about Knowledge of Symptoms Items

\begin{tabular}{lcc}
\hline Items & Correct n (\%) & Incorrect n (\%) \\
\hline Painless tumors, mastitis (like orange) & $48(14.4 \%)$ & $286(85.6 \%)$ \\
Dissimilarity of the size and shape of the breasts since puberty & $78(23.4 \%)$ & $256(76.6 \%)$ \\
Nipple discharge that contains blood & $156(46.7 \%)$ & $178(53.3 \%)$ \\
Inverted Nipple & $118(35.3 \%)$ & $216(64.7 \%)$ \\
Changes in skin breasts & $103(30.8 \%)$ & $231(69.2 \%)$ \\
Repetitive and painful pain before monthly in breasts & $44(13.2 \%)$ & $290(86.8 \%)$ \\
Painless tumors & $158(47.3 \%)$ & $176(52.7 \%)$ \\
\hline
\end{tabular}

that the women knowledge of breast cancer symptoms (repetitive and painful pain before monthly in breasts, painless tumors, and dissimilarity of the size and shape of the breasts since puberty) were very inadequate.

\section{Discussion}

Our study identified predictors of the stage of change for cognitive predictors such as perceived susceptibility, perceived severity, and knowledge of breast cancer symptom to the adoption of breast cancer screening behavior in a sample of Iranian married women. Our finding indicated only $11.7 \%$ of the respondents were in stages of action or maintenance about breast cancer screening adoption. As well as, the findings of the study indicate that the greater proportion of women $(85 \%$ of them) were in the down stages of breast cancer screening adoption (i.e. pre-contemplation and contemplation stages), which may have reflected the relatively lower educational level $(59 \%$ of study participants under diploma educated) of this women as well as their low score of knowledge of breast cancer symptoms (average score of 2.11, ranged from 0 to 7). This finding is not similar to the findings reported by other studies. For example, Wu et al carried out a research on 315 Asian-American women in Michigan and reported $>50 \%$ of women were in the action or maintenance stages of adoption mammography screening behavior (Wu et al., 2009). Furthermore, Strong and Liang (2009) in their study among Chinese American women and indicated largest group among stages was maintenance and $44 \%$ of participants for mammography were in this stage. As well as, Tu et al., (2002) reported that approximately $25 \%$ of the Cambodian American women in their study in the maintenance stage for breast cancer screening behavior. The comparison of our findings with similar researches conducted abroad showed the discrepancy exists between the results and indicates that the stage of breast cancer screening adoption is much lower among Iranian women compared to other countries. Overall, assessing differences stage of breast cancer screening behavior could facilitate the development of stage-matched promotion programs for women ( $\mathrm{Wu}$ et al., 2009). Moreover, breast cancer screening adoption messages must be tailored to women needs in various stages of adopting breast cancer screening behavior. Our findings can be a warning to women health policymakers in Iran and indicated the necessity of development and implementation of planning breast cancer screening promotion programs among Iranian women.
Another finding of our study was the older women had reported in stages of action or maintenance about breast cancer screening behavior much more than younger ones. This finding is similar to the findings reported by other studies (Tung et al., 2008; Barghouti et al., 2008). These results showed that the promotion program must be the focus on younger women because they are may imagine themselves at lower risk of breast cancer.

Our study findings found that there was a significant association between having a family history of breast cancer with breast cancer stage of screening adoption. Consistent with the our study, Tolma er al., (2014) carried out a research on 255 American Indian women from a rural Oklahoma medical clinic and reported women with a family history of breast cancer $(\mathrm{OR}=9.97)$ had a higher odds of past breast cancer screening behavior.

The findings of the present study indicate that the perceived susceptibility and perceived severity of breast cancer, and the knowledge of breast cancer symptoms, were associated with the women breast cancer stage of screening adoption. Perceived susceptibility, perceived severity, and knowledge of breast cancer symptoms were higher in stages of action and maintenance than women in other stages of breast cancer adoption. In the field of women health, several research has underlined the predictive potential of cognitive predictors such as susceptibility, severity, and knowledge, for the adoption of breast cancer screening behaviors (Kim and Menon, 2009; Russell, et al., 2007; Menon, et al., 2007).

One of the important determinants to the adoption of breast cancer screening behavior is knowledge (Alam, 2006). The current study showed that the score of knowledge of breast cancer symptoms was low among subjects. This finding is similar to those reported by other studies conducted among women in the Middle East (Alharbi et al., 2012; Latif 2014; Dandash and Al-Mohaimeed, 2007). For example, Alharbi et al carried out a research on 421 women teachers in Al Jahra, in the west of Kuwait and reported insufficient knowledge about breast cancer among women (Alharbi et al., 2012). As well as, Latif carried out a research with aim of determining knowledge, attitude, and practices about breast cancer in 150 females from a university in Saudi Arabia and reported 106 female of them $(70.7 \%)$ had scored $50.0 \%$ and more, which indicted knowledge of breast cancer symptoms among them was mild (Latif, 2014). Moreover, Dandash and Al-Mohaimeed (2007) in their study stated necessary of designing an educational program to improve the knowledge level of Saudi Arabia women regarding 
breast cancer. These results can be indicated necessary to design an educational program with the aim of increasing women knowledge about breast cancer symptoms. Our cross-sectional study provides useful information that may be utilized by health policymakers for planning effective interventional programs. Our findings indicated that the women knowledge of breast cancer symptoms were inadequate. Thus, planning health promotion programs to rectify the lack of women knowledge of breast cancer symptoms is urgently needed.

The findings reported in this study have certain limitations. First, data collection was based on self-reporting, which is usually prone to recall bias. Second, data collection among sample of married women in the west of Iran and results may be cannot be generalized to all women. The internal consistency of the questionnaire was relatively low $(\alpha=0.63)$ for assessing perceived susceptibility is another limitation of the study.

In conclusion, in order to implement effective breast cancer screening adoption promotion programs, it is critical to understand predictors related to the stage of adoption. Our study focuses on explaining how perceived susceptibility and perceived severity of breast cancer and knowledge of breast cancer symptoms differ across stages of breast cancer screening behavior adoption among the sample of Iranian women. These results can provide better knowledge for the development and implementing of stage-targeted breast cancer screening promotion programs. We conclude that it seems that the focus of health planner should be to rise to perceived severity; perceived susceptibility and knowledge of symptoms of breast cancer about the promotion of breast cancer screening adoption an Iranian woman. Moreover, our findings indicated the need to promote regular breast cancer screening among Iranian women.

\section{Acknowledgements}

We would like to thank all women participants in this study.

\section{Conflict of interest statement}

The authors declare that they have no conflict of interest.

\section{References}

Alam AA (2006). Knowledge of breast cancer and its risk and protective factors among women in Riyadh. Ann Saudi Med, 26, 272-77.

Albert US, Koller M, Wagner U, et al (2004). Survival chances and psychological aspects of quality of life in patients with localized early stage breast cancer. Inflamm Res, 53, 136-41.

Alharbi NA, Alshammari MS, Almutairi BM, et al (2012). Knowledge, awareness, and practices concerning breast cancer among Kuwaiti female school teachers. Alexandria Med $J, 48,75-82$.

Barghouti FF, Takruri AH, Froelicher ES (2008). Awareness and behavior about Pap smear testing in family medicine practice. Saudi Med J, 29, 1036-40.

Dafei M, Dehghani A, Momeni Z, et al (2017). Study of breast cancer knowledge, attitude, and preventive behaviors among women referring to health-treatment centers in Yazd, Iran, 2015. PSJ, 15, 46-53.

Dandash KF, Al-Mohaimeed A (2007). Knowledge, attitudes, and practices surrounding breast cancer and screening in female teachers of Buraidah, Saudi Arabia. Int J Health Sci, 1, 61.

Griva F, Anagnostopoulos F, Gourounti K, et al (2013). Mammography screening within the context of the theory of planned behavior: The role of personality traits and personal factors. Int J Psychol Stud, 5, 98.

Giordano SH, Buzdar AU, Smith TL, et al (2004). Is breast cancer survival improving?. Cancer, 100, 44-52.

Harding C, Pompei F, Burmistrov D, et al (2015). Breast cancer screening, incidence, and mortality across US counties. JAMA Intern Med, 175, 1483-89.

Hosseini SN, Mirzaei-Alavijeh M, Matin BK, et al (2016). Locus of control or self-esteem; which one is the best predictor of academic achievement in Iranian college students. Iran $J$ Psychiatry Behav Sci, 10, e2602.

Ibrahim E, Al Kharousi S, AlBahrani B, et al (2017). A cross-sectional analysis of reproductive risk factors for breast cancer among Omani women. Breast J, 32, 54.

Jalilian F, Karami Matin B, Ahmadpanah M, et al (2014). Substance abuse among college students: Investigation the role of hopelessness. Life Sci J, 11, 396-99.

Kok G, Gottlieb NH, Peters GJ, et al (2016). A taxonomy of behaviour change methods: an intervention mapping approach. Health Psychol Rev, 10, 297-312.

Kim JH, Menon U (2009). Pre- and postintervention differences in acculturation, knowledge, beliefs, and stages of readiness for mammograms among Korean American women. Oncol Nurs Forum, 36, 80-92

Latif R (2014). Knowledge and attitude of Saudi female students towards breast cancer: A cross-sectional study. J Taibah Univ Med Sci, 9, 328-334

Lopez-McKee G (2010). Development of the mammography beliefs and attitudes questionnaire for low-health-literacy Mexican-American women. Online J Issues Nurs, 16, 25-29.

Menon U, Champion V, Monahan PO, et al (2007). Health belief model variables as predictors of progression in stage of mammography adoption. Am J Health Promot, 21, 255-61.

Mirzaei-Alavijeh M, NasirZadeh M, Mostafei M, et al (2011). Anxiety prevalence survey of 144 students from PayamE-Nour Boiene Mieandasht University (Isfahan) and its relationship with irritable bowel syndrome in 2011. Govaresh, 16, 83-90.

Parkin DM, Bray FI, Devesa SS (2001). Cancer burden in the year 2000. The global picture. Eur J Cancer, 37, 4-66.

Pennypacker HS, Naylor L, Sander AA, et al (1999). Why can't we do better breast examinations?. Nurse practitioner, 10, 122-8.

Prochaska JO, Velicer WF (1997). The transtheoretical model of health behavior change. Am J Health Promot, 12, 38-48.

Russell KM, Monahan P, Wagle A, et al (2007). Differences in health and cultural beliefs by stage of mammography screening adoption in African American women. Cancer, 109, 386-95

Royse D, Dignan M (2009). Improving cancer knowledge and screening awareness: test of a telephone interviewer intervention. J Cancer Educ, 24, 315-8.

Saadat S, Yousefifard M, Asady H, et al (2015). The most important causes of death in Iranian population; a Retrospective Cohort Study. Emergency, 3, 16-19.

Spencer L, Pagell F, Adams T (2005). Applying the transtheoretical model to cancer screening behavior. Am J Health Behav, 29, 36-56.

Strong C, Liang W (2009). Relationships between decisional balance and stage of adopting mammography and Pap testing 
among Chinese American women. Cancer Epidemiol, 33, 374-80

Tolma EL, Stoner JA, Li J, et al (2014). Predictors of regular mammography use among American Indian women in Oklahoma: a cross-sectional study. BMC Womens Health, 14, 101.

Tung WC, Nguyen DHT, Tran DN (2008). Applying the transtheoretical model to cervical cancer screening in Vietnamese-American women. Int Nurs Rev, 55, 73-80.

Tu SP, Yasui Y, Kuniyuki A, et al (2002). Breast cancer screening: stages of adoption among Cambodian American women. Cancer Detect Prev, 26, 33-41.

Wang WL, Hsu SD, Wang JH, et al (2014). Survey of breast cancer mammography screening behaviors in Eastern Taiwan based on a health belief model. Kaohsiung J Med Sci, 30, 422-7.

Wu TY, Hsieh HF, West BT (2009). Stages of mammography adoption in Asian American women. Health Educ Res, 24, 748-9.

\section{(c) (i) (9)}

This work is licensed under a Creative Commons AttributionNon Commercial 4.0 International License. 